\title{
Novel investigative modalities for evaluation of pancreatic cystic lesions: Does increased diagnostic accuracy justify the high costs?
}

\section{(ㄷ)(우우}

\author{
Author \\ Somashekar G. Krishna \\ Institution \\ Sections of Pancreatic Disorders and Advanced Endoscopy, \\ Division of Gastroenterology, Hepatology, and Nutrition, \\ The Ohio State University Wexner Medical Center, \\ Columbus, Ohio, United States \\ Bibliography \\ DOI https://doi.org/10.1055/s-0043-119794 | \\ Endoscopy International Open 2017; 05: E996-E998 \\ (c) Georg Thieme Verlag KG Stuttgart · New York \\ ISSN 2364-3722
}

\author{
Corresponding author \\ Somashekar G. Krishna, MD, MPH, Assistant Professor, \\ Sections of Pancreatic disorders and Advanced Endoscopy, \\ 395 W. 12th, Avenue, Suite 262, Division of \\ Gastroenterology, Hepatology and Nutrition, Columbus, \\ Ohio, United States \\ Fax: 614-293-8518 \\ sgkrishna@gmail.com
}

Pancreatic cystic lesions (PCLs) are increasingly detected on imaging studies, resulting in undue anxiety to the patient and the treating physician. Diagnostic differentiation of PCLs continues to be challenging. There have been multiple guidelines for management of PCLs, mostly due to lack of tests with high diagnostic accuracy. Cost-effectiveness studies for managing PCLs have been mostly retrospective and have demonstrated that improvement in diagnostic accuracy effectively prevents unwarranted surgery, thereby reducing healthcare resource utilization. In the last 5 years, molecular analysis of PCL fluid and needle-based confocal laser endomicroscopy (nCLE) have demonstrated promising results with potential to improve diagnostic accuracy of PCLs and thereby impact patient management and outcomes. In a study published in this issue of Endoscopy International Open, Claude Le Pen et al. have performed a cost-effectiveness analysis to evaluate the economic benefit of diagnosing serous cystadenomas (SCA) by augmenting endoscopic ultrasound-guided fine-needle aspiration with nCLE. Despite the increased overall cost of diagnostic evaluation of PCLs, the improved accuracy of diagnosing SCAs and prevention of unwarranted pancreatic surgeries can potentially reduce healthcare resource utilization.

Pancreatic cystic lesions are incidentally diagnosed in $13 \%$ to $45 \%$ of patients undergoing magnetic resonance imaging and $2 \%$ of computed tomography scans [ 1,2$]$. Despite judicious utilization of current standard of care including endoscopic ultrasound (EUS), fine-needle aspiration (FNA), cyst fluid analysis (CEA), and cytology, it is challenging to accurately classify PCLs into mucinous or non-mucinous (serous cystadenomas or pseudocysts) PCLs. In fact, preoperative diagnosis of mucinous PCLs is incorrect in up to $30 \%$ of cases and these benign cysts are often surgically resected, exposing patients to an operative morbidity of $30 \%$ to $40 \%$ [3,4]. Therefore, accurate characterization of PCLs would minimize healthcare costs and resource utilization associated with unwarranted surgical resections.

In a previous cost-effectiveness analysis of the 2006 International Consensus Guidelines (ICG) [5,6], a Markov model was utilized and the authors created 3 scenarios. In Scenario 1, patients with suspected SCAs were followed as a benign disease. In Scenario 2, patients, if deemed fit, underwent surgical resection with no further follow-up. In Scenario 3, patients underwent preoperative EUS-FNA and cyst fluid (CEA and cytology) analysis, and surgery was performed based on the differentiation of PCL [6]. In this analysis, Scenario 2 was the cheapest (US \$13,200 per patient) but with lowest quality-adjusted life years (QALY) and Scenario 3 accounted for the highest costs (\$23,337 per patient) and highest QALY. The cost increment to reach Scenario 3 was $\$ 11,394$, indicating that additional costs were within acceptable range for satisfactory return on investment.

In a more recent cost-effectiveness analysis [7], 4 scenarios were tested in a hypothetical cohort of 1,000 asymptomatic patients with incidental PCLs (size $\geq 3 \mathrm{~cm}$ ). In Scenario 1, wait and watch with cross-sectional imaging, and surgery was recommended for symptoms or high-risk features based on ICG 2012 guidelines [8]. In Scenario 2, patients were considered for resection without initial EUS. In Scenario 3, patients underwent EUS-FNA and cyst fluid analysis (CEA and cytology) and muci- 
nous cysts $(C E A>192 \mathrm{ng} / \mathrm{mL}$ ) were referred for resection. Scenario 4 was similar to Scenario 3 with added cyst fluid integrated molecular pathology (IMP) and surgery was considered for mucinous-aggressive PCLs (other 3 classifications based on IMP, including non-mucinous, mucinous-benign, and mucinous-indolent PCLs, were followed conservatively). Scenario 4 with EUS-FNA-and cyst fluid CEA/cytology with IMP provided the greatest increase in QALY with cost nearly identical to Scenario 1 (\$18,966 vs. $\$ 18,766$ per patient). The authors concluded that adding IMP was the most cost-effective strategy.

Endoscopic ultrasound guided needle-based Confocal Laser Endomicroscopy ( $\mathrm{nCLE}$ ) is an emerging technological advance that provides in vivo, real-time, microscopic imaging of PCLs. More than 500 patients have been enrolled worldwide since 2011 in various studies involving EUS-nCLE for evaluation of PCLs. Recent major trials have established the safety profile and feasibility of diagnostic capabilities of EUS-guided nCLE in patients with PCLs [9-14]. In a recent larger, 11-year retrospective review of surgically resected SCAs at a tertiary US center, only $10 \%$ of SCAs (of 51) were diagnosed preoperatively by EUS-FNA cytology [15]. On histopathology, SCAs demonstrate a dense subepithelial capillary vascular network $[11,16]$. During EUS-nCLE, a characteristic vascular network has been demonstrated and described as "superficial vascular network" or "fern pattern" of vascularity $[11,13,17,18]$. Among external blinded EUS experts, an "almost perfect" inter- and-intraobserver agreement has been demonstrated for identifying the vascular patterns of SCAs (INDEX study) $[13,19]$. Further, the in vivo $\mathrm{nCLE}$ patterns of SCAs have been reproduced in ex vivo CLE of resected PCLs $[17,18]$. While cyst fluid CEA has a pooled sensitivity of $50 \%$ and accuracy of $67 \%$ for diagnosing SCA, cytology has very low diagnostic yield; moreover there is lack of free fluid during FNA from microcystic SCAs [20]. Compared to this, EUS-nCLE has a sensitivity of $69 \%$ and specificity of $100 \%$ for diagnosing SCAs when the characteristic vascular pattern is detected [11].

In this issue of Endoscopy International Open, Claude Le Pen et al. have performed a cost-effectiveness analysis to evaluate the economic benefit of diagnosing SCAs with EUS-FNA and nCLE compared to EUS-FNA alone. They have theorized 2 scenarios based on published studies for patients with indeterminate non-communicating (with main pancreatic duct) PCLs (size $\geq 2 \mathrm{~cm}$ ). Scenario 1 is use of EUS-FNA alone, derived from a retrospective cohort of 2622 patients with a diagnosis of SCA from 1990 to 2014 [21], and Scenario 2 is EUS-FNA with nCLE from a prospective French multicenter study (CONTACT study) [11]. Patients who underwent EUS-FNA with nCLE experienced a $23 \%$ reduction in frequency of surgical interventions, which accounted for $13 \%$ and $14 \%$ reductions in total costs in the public and private sectors, respectively. For every 1,000 patients, 4 did not experience the estimated surgery-associated mortality. In France, the total cost of EUS-FNA with anesthesia was $274.87 €(\$ 397.60)$ and that of nCLE was $600 €(\$ 706.80)$ per patient during the study period. Despite using a maximum nCLE cost estimate of $900 €(\$ 1060.30)$, application of nCLE accounted for cost reductions of approximately $9 \%$ and $7 \%$ for the public and private sectors, respectively.
While we can hypothesize that utilization of EUS-nCLE and cyst fluid molecular analysis will improve diagnostic accuracy for differentiating mucinous from non-mucinous PCLs compared to the current standard of care, there are certain limitations for these novel diagnostic modalities.

Overall, the most significant limitations of studies evaluating EUS-nCLE include: (a) fewer surgically resected lesions in mostly single-center studies; (b) lack of formal training in EUSnCLE; and (c) requirement for high-quality nCLE imaging [12]. Limitations of studies in cyst fluid molecular analysis include: (a) lack of prospective multicenter studies; (b) suboptimal identification of specific PCL types - lower sensitivity for mucinous cystic neoplasm with molecular markers and lack of markers for cystic neuroendocrine tumor and squamous epithelium lined cysts; and (c) lower sensitivity for diagnosing SCAs due to continued dependence on Sanger sequencing for VHL gene [22]. Moreover, there are no studies combining EUS-nCLE and cyst fluid molecular markers in differentiation of PCLs and identification of advanced neoplasia (high-grade dysplasia or cancer) in mucinous-PCLs.

Patients with PCLs are a group with a high risk of operative complications and low risk of developing malignancy. Thus, accurate differentiation of a mucinous from non-mucinous or serous PCL potentially determines a cost-effective strategy. A conclusion from these cost-effectiveness studies indicates that improving diagnostic accuracy of benign non-mucinous PCLs and detecting cysts with malignant potential provides the most benefit in healthcare resource utilization. Because incidence of PCL increases with age, they are more relevant in the elderly population [23]. Despite the evidence provided, well-designed prospective studies are necessary to establish whether the proposed approach of advanced diagnostics is truly cost effective. In the meantime, these novel diagnostic modalities would theoretically result in avoiding unwarranted surgical resections of benign PCLs and promote resection of high-risk mucinous cysts with malignant potential.

\section{Competing interests}

None

References

[1] Moris M, Bridges MD, Pooley RA et al. Association Between Advances in High-Resolution Cross-Section Imaging Technologies and Increase in Prevalence of Pancreatic Cysts From 2005 to 2014. Clin Gastroenterol Hepatol 2016; 14: 585-593 e583

[2] Laffan TA, Horton KM, Klein AP et al. Prevalence of unsuspected pancreatic cysts on MDCT. AJR Am J Roentgenol 2008; 191: 802 - 807

[3] Valsangkar NP, Morales-Oyarvide V, Thayer SP et al. 851 resected cystic tumors of the pancreas: a 33-year experience at the Massachusetts General Hospital. Surgery 2012; 152: S4 - S12

[4] Gaujoux S, Brennan MF, Gonen M et al. Cystic lesions of the pancreas: changes in the presentation and management of 1,424 patients at a single institution over a 15-year time period. Journal of the American College of Surgeons 2011; 212: 590-600; discussion 600-593 
[5] Tanaka M, Chari S, Adsay V et al. International consensus guidelines for management of intraductal papillary mucinous neoplasms and mucinous cystic neoplasms of the pancreas. Pancreatology 2006; 6 : $17-32$

[6] Das A, Ngamruengphong S, Nagendra S et al. Asymptomatic pancreatic cystic neoplasm: a cost-effectiveness analysis of different strategies of management. Gastrointest Endosc 2009; 70: 690 -699 e696

[7] Das A, Brugge W, Mishra G et al. Managing incidental pancreatic cystic neoplasms with integrated molecular pathology is a cost-effective strategy. Endosc Int Open 2015; 3: E479-E486

[8] Tanaka M, Fernandez-del CastilloC, Adsay V et al. International consensus guidelines 2012 for the management of IPMN and MCN of the pancreas. Pancreatology 2012; 12: 183-197

[9] Konda V], Meining A, Jamil LH et al. A pilot study of in vivo identification of pancreatic cystic neoplasms with needle-based confocal laser endomicroscopy under endosonographic guidance. Endoscopy 2013; 45: $1006-1013$

[10] Nakai Y, Iwashita T, Park doH et al. Diagnosis of pancreatic cysts: EUSguided, through-the-needle confocal laser-induced endomicroscopy and cystoscopy trial: DETECT study. Gastrointest Endosc 2015; 81: $1204-1214$

[11] Napoleon B, Lemaistre Al, Pujol B et al. A novel approach to the diagnosis of pancreatic serous cystadenoma: needle-based confocal laser endomicroscopy. Endoscopy 2015; 47: 26-32

[12] Napoleon B, Lemaistre AI, Pujol B et al. In vivo characterization of pancreatic cystic lesions by needle-based confocal laser endomicroscopy (nCLE): proposition of a comprehensive nCLE classification confirmed by an external retrospective evaluation. Surg Endosc 2016; 30: $2603-2612$

[13] Krishna SG, Brugge WR, Dewitt JM. Needle-based confocal laser endomicroscopy for the diagnosis of pancreatic cystic lesions: an international external interobserver and intraobserver study (with videos). Gastrointest Endosc 2017; 86: 644 - 654.e2

[14] Kadayifci A, Atar M, Yang M et al. Imaging of pancreatic cystic lesions with confocal laser endomicroscopy: an ex vivo pilot study. Surg Endosc 2017: doi:10.1007/s00464-017-5577-y [Epub ahead of print]
[15] Lilo MT, VandenBussche C], Allison DB et al. Serous cystadenoma of the pancreas: Potentials and pitfalls of a preoperative cytopathologic diagnosis. Acta Cytol 2017; 61: 27-33

[16] Basturk O, Coban I, Adsay NV. Pancreatic cysts: pathologic classification, differential diagnosis, and clinical implications. Arch Pathol Lab Med 2009; 133: $423-438$

[17] Krishna SG, Modi RM, Kamboj AK et al. In vivo and ex vivo confocal endomicroscopy of pancreatic cystic lesions: A prospective study. World J Gastroenterol 2017; 23: $3338-3348$

[18] Modi RM, Swanson B, Muscarella P et al. Novel techniques for diagnosis of serous cystadenoma: fern pattern of vascularity confirmed by in vivo and ex vivo confocal laser endomicroscopy. Gastrointest Endosc 2017; 85: 258-259

[19] Krishna SG, Swanson B, Hart PA et al. Validation of diagnostic characteristics of needle based confocal laser endomicroscopy in differentiation of pancreatic cystic lesions. Endosc Int Open 2016; 4: E1124E1135

[20] van der Waaij LA, van Dullemen HM, Porte RJ. Cyst fluid analysis in the differential diagnosis of pancreatic cystic lesions: a pooled analysis. Gastrointest Endosc 2005; 62: 383-389

[21] Jais B, Rebours V, Malleo G et al. Serous cystic neoplasm of the pancreas: a multinational study of 2622 patients under the auspices of the International Association of Pancreatology and European Pancreatic Club (European Study Group on Cystic Tumors of the Pancreas). Gut 2016; 65: 305-312

[22] Singhi A, McGrath K, Brand K et al. Prospective DNA Testing of 462 Pancreatic Cysts Reveals the Inclusion of Mutational Allelic Frequencies (AFs) Enhance the Detection of Malignancy in Intraductal Papillary Mucinous Neoplasms (IPMNs). Modern Pathology 2017; 30: $451 \mathrm{~A}-452 \mathrm{~A}$

[23] Huang ES, Gazelle GS, Hur C. Consensus guidelines in the management of branch duct intraductal papillary mucinous neoplasm: a costeffectiveness analysis. Dig Dis Sci 2010; 55: $852-860$ 